\title{
Influence of time delay on bifurcation of a synthetic drug transmission model with psychological addicts
}

\author{
Zizhen Zhang ${ }^{1 *}$, Fangfang Yang ${ }^{1}$ and Wanjun Xia ${ }^{1}$
}

\section{"Correspondence: \\ zzzhaida@163.com \\ 1School of Management Science and Engineering, Anhui University of Finance and Economics, Bengbu, China}

\begin{abstract}
A synthetic drug transmission model with psychological addicts and time delay is proposed in this paper. By analyzing the corresponding characteristic equation and choosing the time delay as the bifurcation parameter, a set of sufficient criteria guaranteeing local stability of the synthetic drug addiction equilibrium and the appearance of a Hopf bifurcation of the model is established. Further, the direction and stability of the Hopf bifurcation are investigated with the aid of normal form theory and center manifold theory. Finally, numerical simulations are performed to support the analytical results.
\end{abstract}

Keywords: Delay; Hopf bifurcation; Synthetic drugs model; Stability; Periodic solution

\section{Introduction}

It is well known that increased use of synthetic drugs such as crystal methamphetamine, ketamine, ecstasy and others, which are a new type of mental drugs based on chemical synthesis is an issue of concern in many parts of the world. Synthetic drugs are becoming increasingly popular in drug markets as they mainly appear in entertainment venues. Taking China for example, the number of reported synthetic drug abusers increased from 1.459 millon in the end of 2014 to 1.538 million in the end of 2017 by the report of China's Drug Situations Report in the past five years (2014-2018) [1]. The number of methamphetamine abusers was up to 1.35 millon which accounted for $56.1 \%$ among the 2.404 million drug-users by the end of 2018 according to China's Drug Situations Report (2018) [2]. What is more serious is that synthetic drugs are more addictive because they can directly affect the central nervous system and show the stronger psychological dependence compared with traditional drugs including heroin, morphine and marijuana, etc. In addition, any drug abuse and dependence constitute one of the most important modes of transmitting human immunodeficiency virus (HIV) and the Hepatitis C virus (HCV) [35]. Obviously, it is urgent to take effective measures to control the spread of synthetic drugs in order to eradicate the tremendous damages and panics brought by synthetic drugs abuse to social and public health system.

(c) The Author(s) 2020. This article is licensed under a Creative Commons Attribution 4.0 International License, which permits use, sharing, adaptation, distribution and reproduction in any medium or format, as long as you give appropriate credit to the original author(s) and the source, provide a link to the Creative Commons licence, and indicate if changes were made. The images or other third party material in this article are included in the article's Creative Commons licence, unless indicated otherwise in a credit line to the material. If material is not included in the article's Creative Commons licence and your intended use is not permitted by statutory regulation or exceeds the permitted use, you will need to obtain permission directly from the copyright holder. To view a copy of this licence, visit http://creativecommons.org/licenses/by/4.0/. 
Since the heroin addiction was first defined as epidemic in 1981-1983 in Ireland, the heroin-using can be modeled in a similar way to the epidemic models [6] and mathematical modeling has been used extensively to address issues of public health importance. For example, Ma et al. developed different forms of heroin epidemic models [3, 6-10] to study the transmission of heroin epidemics. Sharomi et al. formulated different smoking models [11-17] for giving up smoking. There were also some drinking models [18-21] proposed by Mushayabasa et al. to analyze the influence of binge drinking to public health. Similarly, mathematical modeling can be also used to describe the spread of synthetic drugs. In [22, 23], Nyabadza et al. analyzed the methamphetamine transmission in South Africa by constructing a suitable mathematical model. In [24], Liu et al. formulated a synthetic drugs transmission model with treatment and studied global stability and backward bifurcation of the model. Considering the effect of synthetic drugs transmission caused by psychology, Ma et al. [25] proposed the following synthetic drugs transmission model with psychological addicts:

$$
\left\{\begin{array}{l}
\frac{d S(t)}{d t}=A-d S(t)-\beta(N) S(t)(P(t)+H(t)), \\
\frac{d P(t)}{d t}=\beta(N) S(t)(P(t)+H(t))-\pi P(t)-(d+\gamma) P(t), \\
\frac{d H(t)}{d t}=\pi P(t)+\theta T(t)-\sigma H(t)-d H(t), \\
\frac{d T(t)}{d t}=\gamma P(t)+\sigma H(t)-\theta T(t)-d T(t),
\end{array}\right.
$$

in which the total population $(N(t))$ is divided into four classes at time $t$ such as the susceptible $(S(t))$, the psychological addicts $(P(t))$, the physiological addicts $(H(t))$ and the drug-users in treatment $(T(t))$. $A$ is the recruitment rate of the susceptible; $\beta(N)$ is the contact rate; $d$ is the natural death rate of the populations; $\pi, \gamma, \theta$ and $\sigma$ are state transition rates. Ma et al. [25] studied stability of system (1).

As stated in [25], a susceptible one is more likely to initiated drug abuse when he contacts with a physiological addict compared to a psychological addict. On the other hand, there should be a period for a drug user in treatment before he relapses into the physiological addicts due to the effect of treatment and his self-control. With an eye to such considerations and motivated by the work of some other dynamical systems with time delay [26-30], we investigate a more realistic synthetic drugs transmission model as follows:

$$
\left\{\begin{array}{l}
\frac{d S(t)}{d t}=A-d S(t)-\beta_{1} S(t) P(t)-\beta_{2} S(t) H(t), \\
\frac{d P(t)}{d t}=\beta_{1} S(t) P(t)+\beta_{2} S(t) H(t)-\pi P(t)-(d+\gamma) P(t), \\
\frac{d H(t)}{d t}=\pi P(t)+\theta T(t-\tau)-\sigma H(t)-d H(t), \\
\frac{d T(t)}{d t}=\gamma P(t)+\sigma H(t)-\theta T(t-\tau)-d T(t),
\end{array}\right.
$$

where $\beta_{1}$ and $\beta_{2}$ are the contact rates of the psychological addicts and the physiological addicts, respectively. $\tau$ is the time delay due to the period that a drug user in treatment uses before he relapses into the physiological addicts.

We organize this paper as follows: in the following section, we analyze the local stability and appearance of Hopf bifurcation of system (2). Section 3 is concerned with the formulas determining the direction and stability of the Hopf bifurcation. Some numerical simulations are executed to illustrate the analytical results in Sect. 4. Finally, conclusions are presented in Sect. 5. 


\section{Local stability and existence of Hopf bifurcation}

The stability and existence of unique positive equilibrium of system (2) is related to basic reproductive number $\Re_{0}$ on free drug equilibrium point (FEP) $D_{0}$, which is determined with the help of next generation matrix method [31]. The free drug equilibrium point is

$$
D_{0}=\left(\frac{A}{d}, 0,0,0\right)
$$

Consider the following matrices for finding the basic reproductive number $\Re_{0}$ :

$$
\begin{aligned}
& \mathbb{F}=\left(\begin{array}{c}
\beta_{1} S(t) P(t)+\beta_{2} S(t) H(t) \\
0
\end{array}\right), \\
& \mathbb{V}=\left(\begin{array}{c}
\pi P(t)+(d+\gamma) P(t) \\
-\pi P(t)-\theta T(t)+\sigma H(t)+d H(t)
\end{array}\right) .
\end{aligned}
$$

Now the Jacobians of $\mathbb{F}$ and $\mathbb{V}$ at $D_{0}$ are

$$
F=\left(\begin{array}{cc}
\beta_{1} \frac{A}{d} & \beta_{2} \frac{A}{d} \\
0 & 0
\end{array}\right)
$$

and

$$
V=\left(\begin{array}{cc}
b_{0} & 0 \\
-\pi & b_{1}
\end{array}\right)
$$

Here $b_{0}=\pi+d+\gamma, b_{1}=\sigma+d$. The dominant eigenvalue of $F V^{-1}$ represents $\Re_{0}=\rho\left(F V^{-1}\right)$, which is

$$
\Re_{0}=\frac{A\left[\beta_{1}(\sigma+d)+\beta_{2} \pi\right]}{d(\sigma+d)(\pi+\gamma+d)} .
$$

For $\Re_{0}>1$, system (2) has a unique synthetic drug addiction equilibrium $D_{*}\left(S_{*}, P_{*}\right.$, $\left.H_{*}, T_{*}\right)$, where

$$
\begin{aligned}
& S_{*}=\frac{(\pi+d+\gamma) P_{*}}{\beta_{1} P_{*}+\beta_{2} H_{*}}, \quad P_{*}=\frac{\left(\Re_{0}-1\right)+\tilde{L}_{1}}{\left[\left(\beta_{1}+\beta_{2} \tilde{L_{2}}\right) / d\right]}, \\
& H_{*}=\tilde{L} P_{*}, \quad T_{*}=\left[\frac{\gamma}{\theta+d}+\frac{\pi \sigma}{(\theta+d)(\sigma+d)}\right] P_{*} /\left[\frac{d(\theta+\sigma+d)}{(\theta+d)(\sigma+d)}\right],
\end{aligned}
$$

and

$$
\begin{aligned}
& \tilde{L_{1}}=\frac{A \beta_{2} \theta}{\sigma+d}\left[\frac{\gamma}{\theta+d}+\frac{\pi \sigma}{(\theta+d)(\sigma+d)}\right] /\left[\frac{d^{2}(\theta+\sigma+d)(\pi+d+\gamma)}{(\theta+d)(\sigma+d)}\right], \\
& \tilde{L_{2}}=\frac{\pi}{\sigma+d}+\frac{\theta}{\sigma+d}\left[\frac{\gamma}{\theta+d}+\frac{\pi \sigma}{(\theta+d)(\sigma+d)}\right] /\left[\frac{d(\theta+\sigma+d)}{(\theta+d)(\sigma+d)}\right] .
\end{aligned}
$$


At the unique synthetic drug addiction equilibrium $D_{*}\left(S_{*}, P_{*}, H_{*}, T_{*}\right)$, system (2) can be linearized as

$$
\left\{\begin{array}{l}
\frac{d S(t)}{d t}=u_{11} S(t)+u_{12} P(t)+u_{13} H(t), \\
\frac{d P(t)}{d t}=u_{21} S(t)+u_{22} P(t)+u_{23} H(t), \\
\frac{d H(t)}{d t}=u_{32} P(t)+u_{33} H(t)+v_{34} T(t-\tau), \\
\frac{d T(t)}{d t}=u_{42} P(t)+u_{43} H(t)+u_{44} T(t)+v_{44} T(t-\tau),
\end{array}\right.
$$

whose characteristic equation is

$$
\lambda^{4}+U_{3} \lambda^{3}+U_{2} \lambda^{2}+U_{1} \lambda+U_{0}+\left(V_{3} \lambda^{3}+V_{2} \lambda^{2}+V_{1} \lambda+V_{0}\right) e^{-\lambda \tau}=0
$$

with

$$
\begin{aligned}
U_{0}= & u_{33} u_{44}\left(u_{11} u_{22}-u_{12} u_{21}\right)+u_{32} u_{44}\left(u_{13} u_{21}-u_{11} u_{23}\right), \\
U_{1}= & \left(u_{12} u_{21}-u_{11} u_{22}\right)\left(u_{33}+u_{44}\right)-u_{13} u_{21} u_{32}-u_{33} u_{44}\left(u_{11}+u_{22}\right) \\
& +u_{23} u_{32}\left(u_{11}+u_{44}\right), \\
U_{2}= & \left(u_{11}+u_{22}\right)\left(u_{33}+u_{44}\right)-u_{12} u_{21}-u_{23} u_{32}+u_{11} u_{22}+u_{33} u_{44}, \\
U_{3}= & -\left(u_{11}+u_{22}+u_{33}+u_{44}\right), \\
V_{0}= & u_{11} u_{23}\left(u_{42} v_{34}-u_{32} v_{44}\right)+u_{21} v_{34}\left(u_{12} u_{43}-u_{13} u_{42}\right) \\
& +u_{21} v_{44}\left(u_{13} u_{32}-u_{12} u_{33}\right)+u_{11} u_{22}\left(u_{33} v_{44}-u_{43} v_{34}\right), \\
V_{1}= & u_{23}\left(u_{32} v_{44}-u_{42} v_{34}\right)-v_{44}\left(u_{11} u_{22}+u_{11} u_{33}+u_{22} u_{33}\right) \\
& +u_{12} u_{21} v_{44}+\left(u_{11}+u_{22}\right) u_{43} v_{34}, \\
V_{2}= & v_{44}\left(u_{11}+u_{22}+u_{33}\right)-u_{43} v_{34},
\end{aligned}
$$

and

$$
\begin{aligned}
& u_{11}=-\left(d+\beta_{1} P_{*}+\beta_{2} H_{*}\right), \quad u_{12}=-\beta_{1} S_{*}, \quad u_{13}=-\beta_{2} S_{*}, \\
& u_{21}=\beta_{1} P_{*}+\beta_{2} H_{*}, \quad u_{22}=\beta_{1} S_{*}-(\pi+d+\gamma), \quad u_{23}=\beta_{2} S_{*}, \\
& u_{32}=\pi, \quad u_{33}=-(\sigma+d), \quad u_{42}=\gamma, u_{43}=\sigma, \quad u_{44}=-d, \\
& v_{34}=\theta, \quad v_{44}=-\theta .
\end{aligned}
$$

When $\tau=0$, the characteristic equation becomes

$$
\lambda^{4}+U_{03} \lambda^{3}+U_{02} \lambda^{2}+U_{01} \lambda+U_{00}=0
$$

We denote

$$
U_{00}=U_{0}+V_{0}, \quad U_{01}=U_{1}+V_{1}, \quad U_{02}=U_{2}+V_{2}, \quad U_{03}=U_{3}+V_{3} .
$$


and suppose that

$$
\left(C_{1}\right): \quad U_{00}>0, \quad U_{03}>0, \quad U_{02} U_{03}>U_{01}, \quad U_{01} U_{02} U_{03}>U_{00} U_{03}^{2}+U_{01}^{2}
$$

It follows from the Routh-Hurwitz theorem that if the condition $\left(C_{1}\right)$ holds, all roots of Eq. (5) have negative real parts.

For $\tau>0$, assume that $\lambda=i \omega$ with $\omega>0$ is the root of Eq. (4), then we obtain

$$
\left\{\begin{array}{l}
\left(V_{1} \omega-V_{3} \omega^{3}\right) \sin \tau \omega+\left(V_{0}-V_{2} \omega^{2}\right) \cos \tau \omega=U_{2} \omega^{2}-\omega^{4}-U_{0} \\
\left(V_{1} \omega-V_{3} \omega^{3}\right) \cos \tau \omega-\left(V_{0}-V_{2} \omega^{2}\right) \sin \tau \omega=U_{3} \omega^{3}-U_{1} \omega .
\end{array}\right.
$$

This leads to

$$
\omega^{8}+d_{3} \omega^{6}+d_{2} \omega^{4}+d_{1} \omega^{2}+d_{0}=0
$$

with

$$
\begin{aligned}
& d_{0}=U_{0}^{2}-V_{0}^{2}, \quad d_{1}=U_{1}^{2}-2 U_{0} U_{2}-V_{0}^{2}+2 V_{0} V_{2}, \\
& d_{2}=U_{2}^{2}+2 U_{0}-2 U_{1} U_{3}-V_{2}^{2}+2 V_{1} V_{3}, \quad d_{3}=U_{3}^{2}-2 U_{2}-V_{3}^{2} .
\end{aligned}
$$

Letting $\omega^{2}=\chi$, we can rewrite Eq. (7) as follows:

$$
\chi^{4}+d_{3} \chi^{3}+d_{2} \chi^{2}+d_{1} \chi+d_{0}=0
$$

Lemma 1 If $d_{0}<0$ Eq. (8) has at least one positive root.

Suppose that $d_{0} \geq 0$. Based on a similar discussion of the distribution of the roots of Eq. (8) in [32], we define

$$
h(\chi)=\chi^{4}+d_{3} \chi^{3}+d_{2} \chi^{2}+d_{1} \chi+d_{0}=0 .
$$

Then

$$
h^{\prime}(\chi)=4 \chi^{3}+3 d_{3} \chi^{2}+2 d_{2} \chi+d_{1}
$$

Let

$$
4 \chi^{3}+3 d_{3} \chi^{2}+2 d_{2} \chi+d_{1}=0
$$

and $z=\chi+\frac{3 d_{3}}{4}$. Then Eq. (11) becomes

$$
z^{3}+\alpha_{1} z+\beta_{1}=0
$$

with

$$
\alpha_{1}=\frac{d_{2}}{2}-\frac{3}{16} d_{3}^{2}, \quad \beta_{1}=\frac{d_{3}^{3}}{32}-\frac{d_{3} d_{2}}{8}+d_{1} .
$$


Denote

$$
\begin{aligned}
& D=\left(\frac{\beta_{1}}{2}\right)+\left(\frac{\alpha_{1}}{3}\right), \quad v=\frac{-1+\sqrt{3} i}{2}, \\
& z_{1}=\sqrt[3]{-\frac{\beta_{1}}{2}+\sqrt{D}}+\sqrt[3]{-\frac{\beta_{1}}{2}-\sqrt{D}}, \\
& z_{2}=\sqrt[3]{-\frac{\beta_{1}}{2}+\sqrt{D}} v+\sqrt[3]{-\frac{\beta_{1}}{2}-\sqrt{D}} v^{2}, \\
& z_{3}=\sqrt[3]{-\frac{\beta_{1}}{2}+\sqrt{D}} v^{2}+\sqrt[3]{-\frac{\beta_{1}}{2}-\sqrt{D}} v, \\
& \chi_{i}=z_{i}-\frac{3 d_{3}}{4}, \quad i=1,2,3 .
\end{aligned}
$$

Lemma 2 If $D \geq 0$, then Eq. (8) has positive roots if and only if $\chi_{1}>0$ and $h\left(\chi_{1}\right)<0$; if $D<0$, then Eq. (8) has positive roots if and only if there exists at least one $\chi^{*} \in\left\{\chi_{1}, \chi_{2}, \chi_{3}\right\}$, such that $\chi^{*}>0$ and $h\left(\chi^{*}\right) \leq 0$.

Thus, if the condition $\left(C_{2}\right)$ holds: (i) $d_{0}<0$ or (ii) $d_{0} \geq 0, D \geq 0, \chi_{1}>0$ and $h\left(z_{1}\right) \leq 0$ or (iii) $d_{0} \geq 0, D<0$, and there exists a $\chi^{*} \in\left\{\chi_{1}, \chi_{2}, \chi_{3}\right\}$, such that $\chi^{*}>0$ and $h\left(\chi^{*}\right) \leq 0$, then Eq. (8) has at least one positive root.

Without loss of generality, we assume that Eq. (8) has four positive roots, denoted by $\chi_{1}$, $\chi_{2}, \chi_{3}$ and $\chi_{4}$. Then Eq. (7) has a positive root $\omega_{i}=\sqrt{\chi_{i}}, i=1,2,3,4$. From Eq. (6), we have

$$
\tau_{i}^{j}= \begin{cases}\frac{1}{\omega_{i}} \times \arccos \left\{\frac{l_{7} \omega_{i}^{6}+l_{6} \omega_{i}^{4}+l_{5} \omega_{i}^{2}+l_{4}}{l_{3} \omega_{i}^{6}+l_{2} \omega_{i}^{4}+l_{1} \omega_{i}^{2}+l_{0}}\right\}+2 j \pi, & \sin \left(\tau_{i} \omega_{i}\right) \geq 0, \\ \frac{1}{\omega_{i}} \times\left[2 \pi-\arccos \left\{\frac{l_{7} \omega_{i}^{6}+l_{6} \omega_{i}^{4}+l_{5} \omega_{i}^{2}+l_{4}}{l_{3} \omega_{i}^{6}+l_{2} \omega_{i}^{4}+l_{1} \omega_{i}^{2}+l_{0}}\right\}\right]+2 j \pi, & \sin \left(\tau_{i} \omega_{i}\right)<0 .\end{cases}
$$

with $i=1,2,3,4, j=0,1,2, \ldots$, and

$$
\begin{aligned}
& l_{0}=V_{0}^{2}, \quad l_{1}=V_{1}^{2}-2 V_{0} V_{2}, \quad l_{2}=V_{2}^{2}-2 V_{1} V_{3}, \quad l_{3}=V_{3}^{2}, \quad l_{4}=-U_{0} V_{0}, \\
& l_{5}=U_{0} V_{2}+U_{2} V_{0}-U_{1} V_{1}, \quad l_{6}=U_{1} V_{3}+U_{3} V_{1}-U_{2} V_{2}-V_{0}, \quad l_{7}=V_{2}-U_{3} V_{3} .
\end{aligned}
$$

Define

$$
\tau_{0}=\min \left\{\tau_{i}^{0} \mid i=1,2,3,4\right\}
$$

such that Eq. (4) has a pair of purely imaginary roots $\omega_{0}$ when $\tau=\tau_{0}$.

Next, we examine the transversality condition. Differentiating Eq. (4) with regard to $\tau$, we obtain the derivative of $\tau$ as follows:

$$
\left[\frac{d \lambda}{d \tau}\right]^{-1}=-\frac{4 \lambda^{4}+3 U_{3} \lambda^{2}+2 U_{2} \lambda+U_{1}}{\lambda\left(\lambda^{4}+U_{3} \lambda^{3}+U_{2} \lambda^{2}+U_{1} \lambda+U_{0}\right)}+\frac{3 V_{3} \lambda^{2}+2 V_{2} \lambda+V_{1}}{\lambda\left(V_{3} \lambda^{3}+V_{2} \lambda^{2}+V_{1} \lambda+V_{0}\right)}-\frac{\lambda}{\tau} .
$$

Replacing $\lambda$ by $i \omega_{0}$, we obtain

$$
\left[\frac{d \lambda}{d \tau}\right]_{\lambda=i \omega_{0}}^{-1}=\frac{h^{\prime}\left(\chi_{0}\right)}{l_{3} \omega_{0}^{6}+l_{2} \omega_{0}^{4}+l_{1} \omega_{0}^{2}+l_{0}},
$$

where $h(\chi)=\chi^{4}+d_{3} \chi^{3}+d_{2} \chi^{2}+d_{1} \chi+d_{0}$ and $\chi_{0}=\omega_{0}^{2}$. 
Therefore, if the condition $\left(C_{3}\right): h^{\prime}\left(\chi_{0}\right) \neq 0$ holds, then the transversality condition is satisfied. Based on the discussion above and the fundamental Hopf bifurcation theorem in [33], we are able to formulate the following theorem.

Theorem 1 For system (2), if the conditions $\left(C_{1}\right)-\left(C_{3}\right)$ hold, then the synthetic drug addiction equilibrium $D_{*}\left(S_{*}, P_{*}, H_{*}, T_{*}\right)$ is locally asymptotically stable when $\tau_{\epsilon}\left[0, \tau_{0}\right)$; system (2) undergoes a Hopf bifurcation at the synthetic drug addiction equilibrium $D_{*}\left(S_{*}, P_{*}, H_{*}, T_{*}\right)$ when $\tau=\tau_{0}$ and a family of periodic solutions bifurcate from the synthetic drug addiction equilibrium $D_{*}\left(S_{*}, P_{*}, H_{*}, T_{*}\right)$.

\section{Direction and stability of Hopf bifurcation}

In this section, direction and stability of Hopf bifurcation will be studied by using center manifold theorem and normal form theory introduced by Hassard et al. [33]. Let $\tau=\tau_{0}+$ $\mu, \mu \in R, u_{1}(t)=S(\tau t), u_{2}(t)=P(\tau t), u_{3}(t)=H(\tau t)$ and $u_{4}(t)=T(\tau t)$. System (2) can be written in a functional differential equation in $C=C\left([-1,0], R^{4}\right)$ as follows:

$$
\dot{u}(t)=L_{\mu}\left(u_{t}\right)+F\left(\mu, u_{t}\right)
$$

where $u(t+\theta)=u_{t}(\theta), L_{\mu}: C \rightarrow R^{4}$ and $F: R \times C \rightarrow R^{4}$,

$$
\begin{aligned}
& L_{\mu} \phi=\left(\tau_{0}+\mu\right)(U \phi(0)+V \phi(-1)), \\
& F(\mu, \phi)=\left(\begin{array}{c}
-\beta_{1} \phi_{1}(0) \phi_{2}(0)-\beta_{2} \phi_{1}(0) \phi_{3}(0) \\
\beta_{1} \phi_{1}(0) \phi_{2}(0)+\beta_{2} \phi_{1}(0) \phi_{3}(0) \\
0 \\
0
\end{array}\right), \\
& U_{\text {trix }}=\left(\begin{array}{cccc}
u_{11} & u_{12} & u_{13} & 0 \\
u_{21} & u_{22} & u_{23} & 0 \\
0 & u_{32} & u_{33} & 0 \\
0 & u_{42} & u_{43} & u_{44}
\end{array}\right), \quad V_{\text {trix }}=\left(\begin{array}{cccc}
0 & 0 & 0 & 0 \\
0 & 0 & 0 & 0 \\
0 & 0 & 0 & v_{34} \\
0 & 0 & 0 & v_{44}
\end{array}\right) .
\end{aligned}
$$

By using the Riesz representation theorem, let $\eta(\theta, \mu):[-1,0] \rightarrow R^{4 \times 4}$ be a function of bounded variation. For $\phi \in C[-1,0]$, let

$$
L_{\mu} \phi=\int_{-1}^{0} d \eta(\theta, \mu) \phi(\theta)
$$

Moreover, we can choose

$$
\eta(\theta, \mu)=\left(\tau_{0}+\mu\right)\left(U_{\text {trix }} \delta(\theta)+V_{\text {trix }} \delta(\theta+1)\right),
$$

where $\delta(\theta)$ is the Dirac delta function.

Define

$$
A(\mu) \phi= \begin{cases}\frac{d \phi(\theta)}{d \theta}, & -1 \leq \theta<0 \\ \int_{-1}^{0} d \eta(\theta, \varrho) \phi(\theta), & \theta=0\end{cases}
$$


and

$$
R(\mu) \phi= \begin{cases}0, & -1 \leq \theta<0 \\ F(\mu, \phi), & \theta=0\end{cases}
$$

where $\phi \in C\left([-1,0], R^{4}\right)$. Then system (18) is equivalent to

$$
\dot{u}(t)=A(\mu) u_{t}+R(\mu) u_{t} .
$$

For $\varphi \in C^{1}\left([0,1],\left(R^{4}\right)^{*}\right)$, define the adjoint operator of $A(0)$ as follows:

$$
A^{*}(\varphi)= \begin{cases}-\frac{d \varphi(s)}{d s}, & 0<s \leq 1 \\ \int_{-1}^{0} d \eta^{T}(s, 0) \varphi(-s), & s=0\end{cases}
$$

and the inner product form

$$
\langle\varphi(s), \phi(\theta)\rangle=\bar{\varphi}(0) \phi(0)-\int_{\theta=-1}^{0} \int_{\xi=0}^{\theta} \bar{\varphi}(\xi-\theta) d \eta(\theta) \phi(\xi) d \xi \text {, }
$$

with $\eta(\theta)=\eta(\theta, 0)$.

Let $g(\theta)=\left(1, g_{2}, g_{3}, g_{4}\right)^{T} e^{i \tau_{0} \omega_{0} \theta}$ denote a eigenvector of $A(0)$ and $+i \tau_{0} \omega_{0}$ is the associated eigenvalue; $\tilde{g}^{*}(\theta)=\tilde{G}\left(1, g_{2}^{*}, g_{3}^{*}, g_{4}^{*}\right)^{T} e^{i \tau_{0} \omega_{0} \theta}$ denotes a eigenvector of $A^{*}$ and $-i \tau_{0} \omega_{0}$ is the associated eigenvalue. Then we obtain

$$
\begin{aligned}
& g_{2}=\frac{u_{23}\left(i \omega_{0}-u_{11}\right)+u_{13} u_{21}}{u_{12} u_{23}+u_{13}\left(i \omega_{0}-u_{22}\right)}, \\
& g_{3}=\frac{\left(i \omega_{0}-u_{22}\right) g_{2}-u_{21}}{u_{23}}, \\
& g_{4}=\frac{u_{42} g_{2}+u_{43} g_{3}}{i \omega_{0}-u_{44}-v_{44} e^{-i \tau_{0} \omega_{0}}}, \\
& g_{2}^{*}=-\frac{i \omega_{0}+u_{11}}{u_{21}}, \\
& g_{3}^{*}=\frac{\left[u_{43}\left(i \omega_{0}+u_{22}\right)-u_{23} u_{42}\right] \tilde{g}_{2}^{*}+u_{12} u_{43}-u_{13} u_{42}}{u_{42}\left(i \omega_{0}+u_{33}\right)-u_{32} u_{43}}, \\
& g_{4}^{*}=-\frac{v_{34} e^{i \tau_{0} \omega_{0}} \tilde{g}_{3}^{*}}{i \omega_{0}+u_{44}+v_{44} e^{i \tau_{0} \omega_{0}}} .
\end{aligned}
$$

From Eq. (27), we can obtain

$$
\left\langle g^{*}, g\right\rangle=\bar{G}\left[1+g_{2} \bar{g}_{2}^{*}+g_{3} \bar{g}_{3}^{*}+g_{4} \bar{g}_{4}^{*}+\tau_{0} e^{-i \tau_{0} \omega_{0}} g_{4}\left(v_{34} g_{3}^{*}+v_{44} g_{4}\right)\right] \text {. }
$$

Let $\left\langle g^{*}, g\right\rangle=1$, then

$$
\bar{G}=\left[1+g_{2} \bar{g}_{2}^{*}+g_{3} \bar{g}_{3}^{*}+g_{4} \bar{g}_{4}^{*}+\tau_{0} e^{-i \tau_{0} \omega_{0}} g_{4}\left(v_{34} g_{3}^{*}+v_{44} g_{4}\right)\right]^{-1}
$$

In the following part of this section, we use the similar computation process as that in [34-38] and then obtain the expressions of $g_{20}, g_{11}, g_{02}$ and $g_{21}$ :

$$
g_{20}=2 \bar{G} \tau_{0}\left(\bar{g}_{2}^{*}-1\right)\left(\beta_{1} g_{2}+\beta_{2} g_{3}\right)
$$



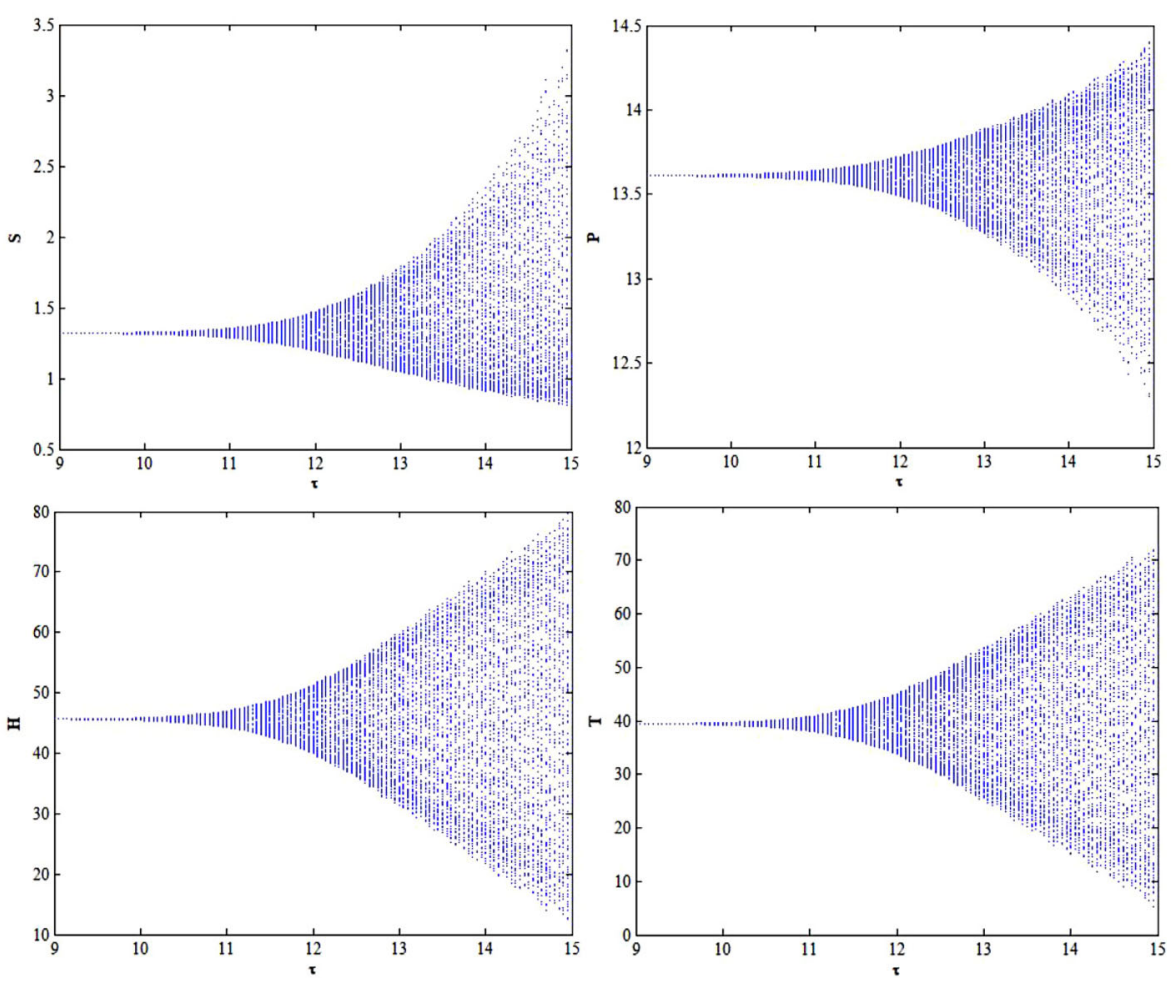

Figure 1 The bifurcation diagram with respect to $\tau$

$$
\begin{aligned}
g_{11}= & \bar{G} \tau_{0}\left(\bar{g}_{2}^{*}-1\right)\left[\beta_{1}\left(g_{2}+\bar{g}_{2}\right)+\beta_{2}\left(g_{3}+\bar{g}_{3}\right)\right], \\
g_{02}= & 2 \bar{G} \tau_{0}\left(\bar{g}_{2}^{*}-1\right)\left(\beta_{1} \bar{g}_{2}+\beta_{2} \bar{g}_{3}\right), \\
g_{21}= & 2 \bar{G} \tau_{0}\left(\bar{g}_{2}^{*}-1\right)\left[\beta_{1}\left(W_{11}^{(1)}(0) g_{2}+\frac{1}{2} W_{20}^{(1)}(0) \bar{g}_{2}+W_{11}^{(2)}(0)+\frac{1}{2} W_{20}^{(2)}(0)\right)\right. \\
& \left.+\beta_{2}\left(W_{11}^{(1)}(0) g_{3}+\frac{1}{2} W_{20}^{(1)}(0) \bar{g}_{3}+W_{11}^{(3)}(0)+\frac{1}{2} W_{20}^{(3)}(0)\right)\right],
\end{aligned}
$$

with

$$
\begin{aligned}
& W_{20}(\theta)=\frac{i g_{20} g(0)}{\tau_{0} \omega_{0}} e^{i \tau_{0} \omega_{0} \theta}+\frac{i \bar{g}_{02} \bar{g}(0)}{3 \tau_{0} \omega_{0}} e^{-i \tau_{0} \omega_{0} \theta}+E_{1} e^{2 i \tau_{0} \omega_{0} \theta}, \\
& W_{11}(\theta)=-\frac{i g_{11} g(0)}{\tau_{0} \omega_{0}} e^{i \tau_{0} \omega_{0} \theta}+\frac{i \bar{g}_{11} \bar{g}(0)}{\tau_{0} \omega_{0}} e^{-i \tau_{0} \omega_{0} \theta}+E_{2} .
\end{aligned}
$$

$E_{1}$ and $E_{2}$ can be obtained by the following two equations:

$$
\begin{aligned}
E_{1}= & 2\left(\begin{array}{cccc}
2 i \omega_{0}-u_{11} & -u_{12} & -u_{13} & 0 \\
-u_{21} & 2 i \omega_{0}-u_{22} & -u_{23} & 0 \\
0 & -u_{32} & 2 i \omega_{0}-u_{33} & -v_{34} e^{-2 i \tau_{0} \omega_{0}} \\
0 & -u_{42} & -u_{43} & 2 i \omega_{0}-u_{44}-v_{44} e^{-2 i \tau_{0} \omega_{0}}
\end{array}\right)^{-1} \\
& \times\left(\begin{array}{c}
-\beta_{1} g_{2}-\beta_{2} g_{3} \\
\beta_{1} g_{2}+\beta_{2} g_{3} \\
0 \\
0
\end{array}\right)
\end{aligned}
$$




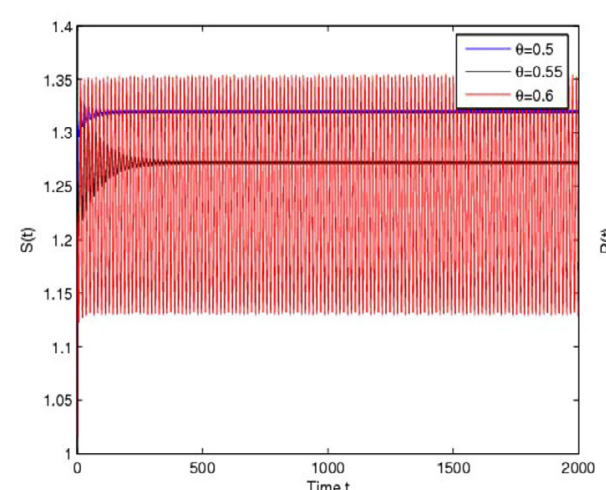

(a)

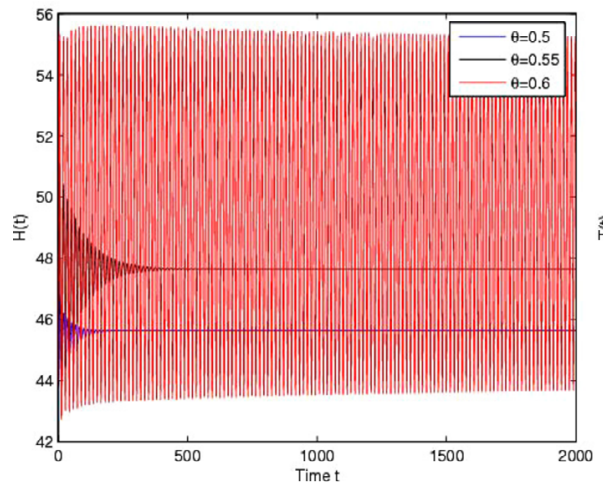

(c)

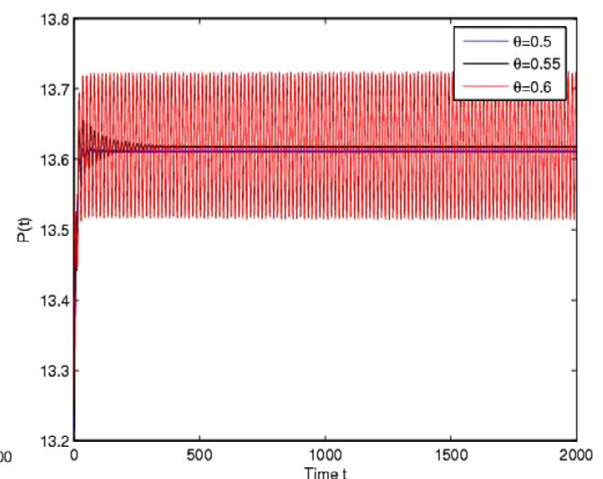

(b)

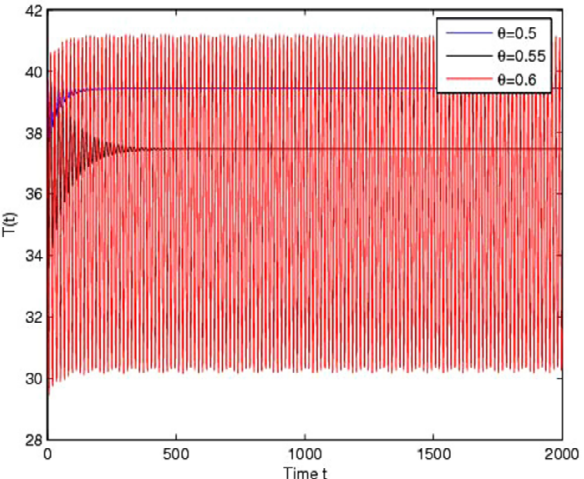

(d)

Figure 2 Time plots of $S, P, H$ and $T$ for different $\theta$ at $\tau=5.835$. The remaining parameters are taken as given in the text

$$
E_{2}=\left(\begin{array}{cccc}
u_{11} & u_{12} & u_{13} & 0 \\
u_{21} & u_{22} & u_{23} & 0 \\
0 & u_{32} & u_{33} & v_{34} \\
0 & u_{42} & u_{43} & u_{44}+v_{44}
\end{array}\right)^{-1} \times\left(\begin{array}{c}
-\beta_{1}\left(g_{2}+\bar{g}_{2}\right)-\beta_{2}\left(g_{3}+\bar{g}_{3}\right) \\
\beta_{1}\left(g_{2}+\bar{g}_{2}\right)+\beta_{2}\left(g_{3}+\bar{g}_{3}\right) \\
0 \\
0
\end{array}\right) .
$$

Then we obtain

$$
\begin{aligned}
& C_{1}(0)=\frac{i}{2 \tau_{0} \omega_{0}}\left(g_{11} g_{20}-2\left|g_{11}\right|^{2}-\frac{\left|g_{02}\right|^{2}}{3}\right)+\frac{g_{21}}{2}, \\
& \mu_{2}=-\frac{\operatorname{Re}\left\{C_{1}(0)\right\}}{\operatorname{Re}\left\{\lambda^{\prime}\left(\tau_{0}\right)\right\}} \\
& \beta_{2}=2 \operatorname{Re}\left\{C_{1}(0)\right\}, \\
& T_{2}=-\frac{\operatorname{Im}\left\{C_{1}(0)\right\}+\mu_{2} \operatorname{Im}\left\{\lambda^{\prime}\left(\tau_{0}\right)\right\}}{\tau_{0} \omega_{0}} .
\end{aligned}
$$

From the above formulas, we can conclude as follows.

Theorem 2 For system (2), the following results hold. If $\mu_{2}>0\left(\mu_{2}<0\right)$, then the Hopf bifurcation is supercritical (subcritical); if $\beta_{2}<0\left(\beta_{2}>0\right)$, then the bifurcating periodic solutions are stable (unstable); if $T_{2}>0\left(T_{2}<0\right)$, then the period of the bifurcating periodic solutions increase (decrease). 


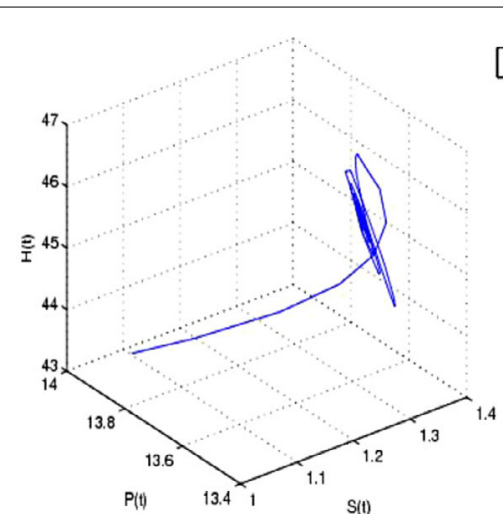

(a)

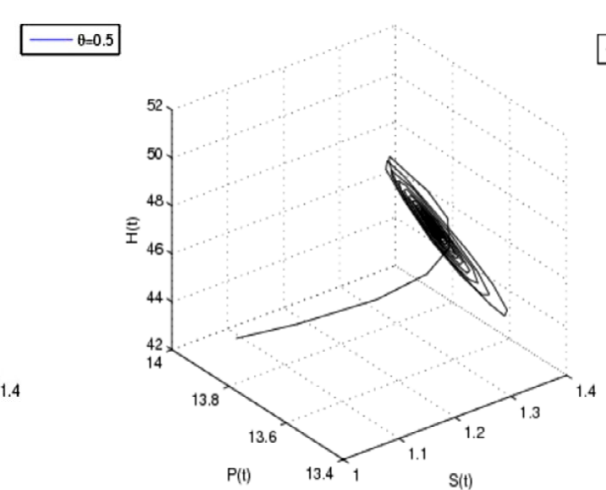

(b)

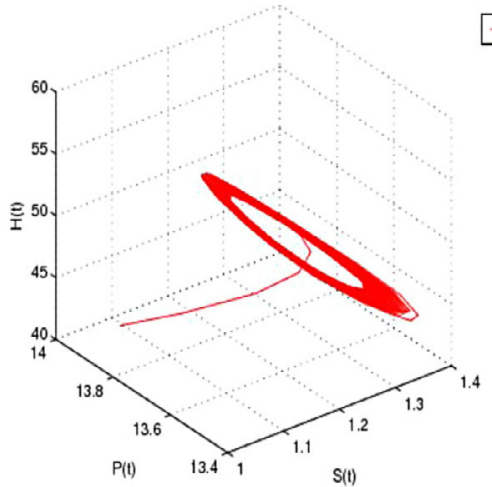

(c)

Figure 3 The phase plots of system (33) for different $\theta$ at $\tau=5.7850$. The remaining parameters are taken as given in the text

\section{Numerical simulations}

In this section, we carry out computer simulations using the Matlab software package to illustrate the obtained theoretical results. Taking $A=2, d=0.02, \beta_{1}=0.016, \beta_{2}=0.028$, $\pi=0.03, \gamma=0.095, \theta=0.5$ and $\sigma=0.421$, we obtain the following specific case of system (2):

$$
\left\{\begin{array}{l}
\frac{d S(t)}{d t}=2-0.02 S(t)-0.016 S(t) P(t)-0.028 S(t) H(t), \\
\frac{d P(t)}{d t}=0.016 S(t) P(t)+0.028 S(t) H(t)-0.145 P(t), \\
\frac{d H(t)}{d t}=0.03 P(t)+0.5 T(t-\tau)-0.441 H(t), \\
\frac{d T(t)}{d t}=0.095 P(t)+0.421 H(t)-0.5 T(t-\tau)-0.02 T(t) .
\end{array}\right.
$$

Then one can obtain $\Re_{0}=12.3481>1$ and the unique synthetic drug addiction equilibrium $D_{*}(1.3196,13.6111,45.6355,39.4338)$ of system (33). Through some calculations, Eq. (8) becomes

$$
\chi^{4}+0.0095 \chi^{3}+1.0218 \chi^{2}-0.2006 \chi-3.21=0 .
$$

Thus, it is easy to see that Eq. (34) has one positive root $\chi_{0}=1.1885$. Then we obtain $\omega_{0}=1.0902$ and $\tau_{0}=9.7367, h^{\prime}\left(\chi_{0}\right)=7.7692>0$. According to Theorem 1, we can con- 


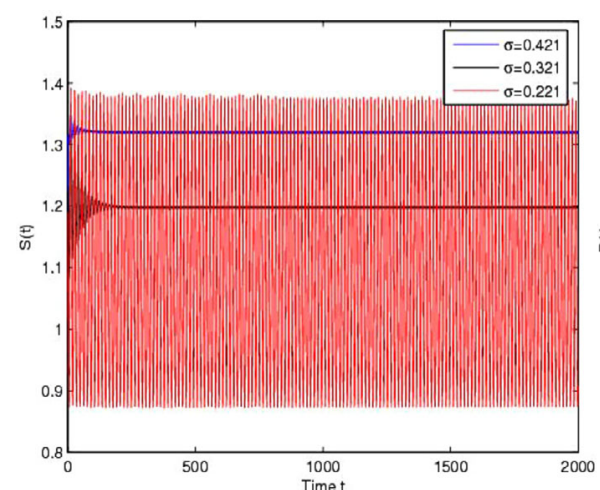

(a)

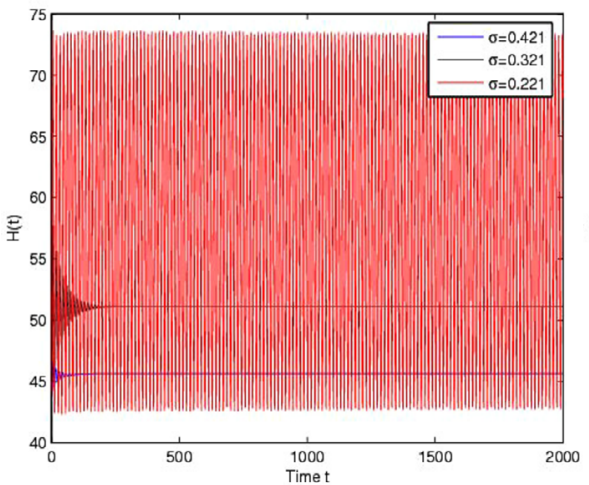

(b)

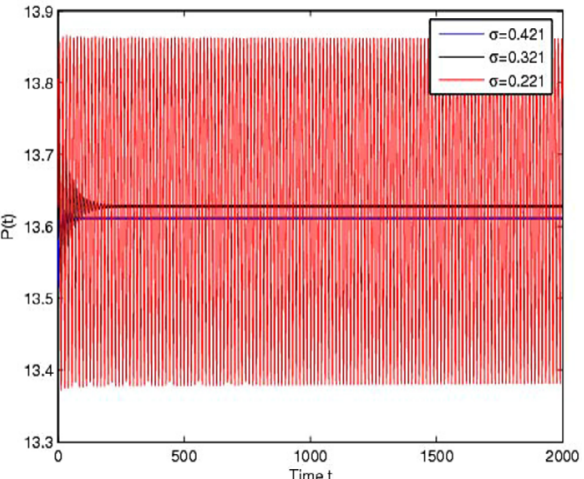

(b)

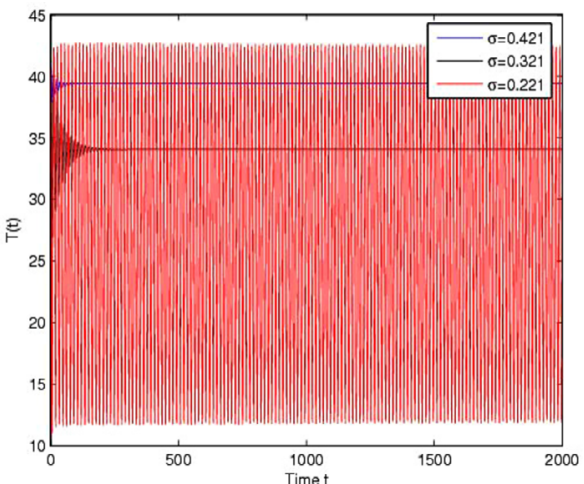

(d)

Figure 4 Time plots of $S, P, H$ and $T$ for different $\sigma$ at $\tau=4.738$. The remaining parameters are taken as given in the text

clude that the synthetic drug addiction equilibrium $D_{*}(1.3196,13.6111,45.6355,39.4338)$ of system (33) is locally asymptotically stable when $\tau \in[0,9.7367)$, and a Hopf bifurcation appears when the value of $\tau$ passes through the critical value 9.7367, which can be indicated by bifurcation diagrams in Fig. 1. Furthermore, based on Eq. (32), $\mu_{2}=1.5613>0$, $\beta_{2}=-2.07<0$ and $T_{2}=0.3611>0$ can be obtained. Accordingly, through Theorem 2, it can be concluded that the Hopf bifurcation at $\tau_{0}$ is supercritical; the bifurcating periodic solutions are stable and increasing.

In addition, Fig. 2 manifests that the numbers of susceptibles and drug-users in treatment decrease, whereas the numbers of the psychological and physiological addicts increase as we increase $\theta$ from 0.5 to 0.6 . Also, due to the increase in $\theta$ from 0.5 to 0.6 , system (33) loses its stability and shows limit cycle behavior, which is illustrated in Fig. 3. It is interesting to note that Fig. 4 reveals the reverse effect of increasing $\sigma$ in a particular range on the four populations in system (33). Figure 5 shows that system (33) changes its behavior from limit cycle to stable focus as we increase the value of $\sigma$.

\section{Conclusions}

The prevention and control of synthetic drugs have received great attention due to their increasingly serious consequences on population. Quite a few mathematical models describing synthetic drugs transmission have been put up by scholars at home and abroad in 


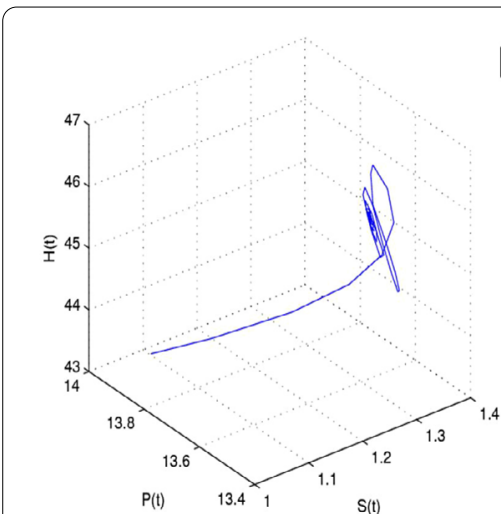

(a)

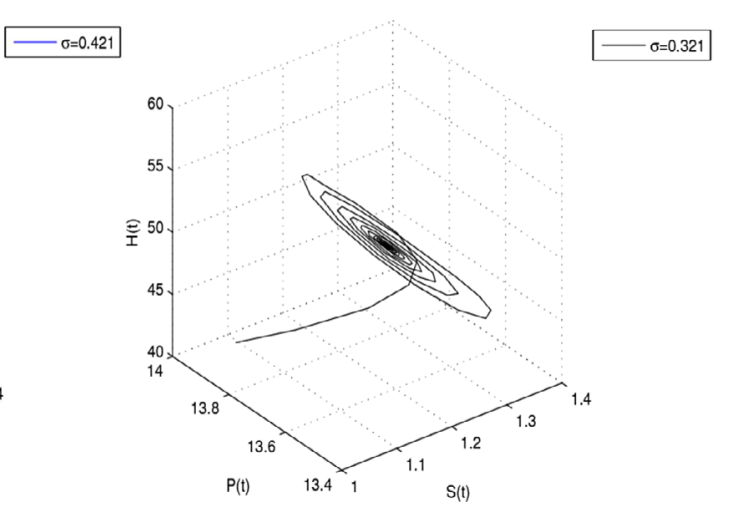

(b)

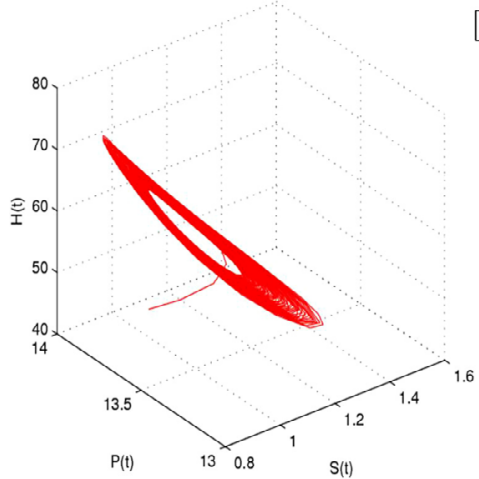

(c)

Figure 5 The phase plots of system (33) for different $\sigma$ at $\tau=4.6805$. The remaining parameters are taken as given in the text

recent years. However, these models neglect the time delay during the process of synthetic drugs transmission. In the present article, synthetic drugs transmission model with time delay is established based on the work in [25]. A series of sufficient criteria guaranteeing the stability and appearance of Hopf bifurcation for the established model are derived by analyzing the corresponding characteristic equation. Moreover, the properties of the Hopf bifurcation are investigated by using the center manifold theorem and normal form theory.

The study shows that the time delay has an important impact on the stability and Hopf bifurcation of the model. When the value of the time delay is below the critical value $\tau_{0}$, synthetic drugs transmission can be predicted and controlled. Contrarily, synthetic drugs transmission will be out of control once the value of the time delay passes through the critical value. Also, we observed that the change of the parameters of $\theta$ and $\sigma$ in a particular range can affect the dynamics of the system based on numerical simulations. Since the numbers of the psychological and physiological addicts increase with the increase of $\theta$ but with the decrease of $\sigma$, it is strongly suggested that drug-users should have strong will once they decide to give up drugs. On the other hand, any person who has been contaminated with drugs should go to the regular detoxification center in time for treatment. 


\section{Acknowledgements}

The authors are grateful to the editor and the anonymous referees for their valuable comments and suggestions on the paper.

\section{Funding}

This research was supported by Project of Support Program for Excellent Youth Talent in Colleges and Universities of Anhui Province (No. gxyqZD2018044) and the Natural Science Foundation of the Higher Education Institutions of Anhui Province (Nos. KJ2019A0655, KJ2019A0656, KJ2019A0662, KJ2020A0002, KJ2020A0014, KJ2020A0016).

\section{Availability of data and materials}

All of the authors declare that all the data can be accessed in our manuscript in the numerical simulation section.

\section{Competing interests}

The authors declare that there is no conflict of interests.

\section{Authors' contributions}

All authors read and approved the final manuscript.

\section{Publisher's Note}

Springer Nature remains neutral with regard to jurisdictional claims in published maps and institutional affiliations.

Received: 21 December 2019 Accepted: 25 March 2020 Published online: 01 April 2020

\section{References}

1. China's Drug Situations Report in past five years (2014-2018). http://www.sohu.com/a/321547191_120024602 (accessed on 20 October 2019)

2. China's Drug Situations Report (2018). http://www.nncc626.com/2019-06/17/c_1210161797.htm (accessed on 20 October 2019)

3. Liu, S.T., Zhang, L., Xing, Y.F.: Dynamics of a stochastic heroin epidemic model. J. Comput. Appl. Math. 351, 260-269 (2019)

4. Garten, R., Lai, S., Zhang, J.: Rapid transmission of hepatitis C virus among young injecting heroin users in southern China. Int. J. Epidemiol. 33, 182-188 (2004)

5. Glatt, S., Su, J., Zhu, S.: Genome-wide linkage analysis of heroin dependence in Han Chinese: results from wave one of a multi-stage study. Am. J. Med. Genet., Part B Neuropsychiatr. Genet. 141, 648-652 (2006)

6. Ma, M.J., Liu, S.Y., Li, J.: Bifurcation of a heroin model with nonlinear incidence rate. Nonlinear Dyn. 88, 555-565 (2017)

7. White, E., Comiskey, C.: Heroin epidemics, treatment and ODE modelling. Math. Biosci. 208, 312-324 (2007)

8. Wang, X.Y., Yang, J.Y., Li, X.Z.: Dynamics of a heroin epidemic model with very population. Appl. Math. 2, 732-738 (2011)

9. Wangari, I.M., Stone, L.: Analysis of a heroin epidemic model with saturated treatment function. J. Appl. Math. 2017, Article ID $1953036(2017)$

10. Wei, Y.C., Yang, Q.G., Li, G.J.: Dynamics of the stochastically perturbed heroin epidemic model under non-degenerate noises. Physica A 526, Article ID 120914 (2019)

11. Sharomi, O., Gumel, A.B.: Curtailing smoking dynamics: a mathematical modeling approach. Appl. Math. Comput. 195, 475-499 (2008)

12. Zaman, G.: Qualitative behavior of giving up smoking models. Bull. Malays. Math. Soc. 34, 403-415 (2011)

13. Singh, J., Kumar, D., Qurashi, M.A., Baleanu, D.: A new fractional model for giving up smoking dynamics. Adv. Differ. Equ. 2017, Article ID 88 (2017)

14. Zeb, A., Zaman, G., Momani, S.: Square-root dynamics of a giving up smoking model. Appl. Math. Model. 37 5326-5334 (2013)

15. Din, Q., Ozair, M., Hussain, T., Saeed, U.: Qulitative behavior of a smoking model. Adv. Differ. Equ. 2016, Article ID 96 (2016)

16. Zhang, X.K., Zhang, Z.Z., Tong, J.Y., Dong, M.: Ergodicity of stochastic smoking model and parameter estimation. Adv. Differ. Equ. 2016, Article ID 274 (2016)

17. Rahman, G., Agarwal, R.P., Liu, L.L., Khan, A.: Threshold dynamics and optimal control of an age-structured giving up smoking model. Nonlinear Anal., Real World Appl. 43, 96-120 (2018)

18. Mushayabasa, S., Bhunu, C.P.: Modelling the effects of heavy alcohol consumption on the transmission dynamics of gonorrhea. Nonlinear Dyn. 66, 695-706 (2011)

19. Xiang, H., Zhu, C.C., Huo, H.F.: Global stability of a drinking model with relapse. J. Biomath. 31, 15-27 (2016)

20. Huo, H.F., Chen, Y.L., Xiang, H.: Stability of a binge drinking model with delay. J. Biol. Dyn. 11, 210-225 (2017)

21. Huo, H.F., Jing, S.L., Wang, X.Y., Xiang, H.: Modelling and analysis of an alcoholism model with treatment and effect of Twitter. Math. Biosci. Eng. 16, 3595-3622 (2019)

22. Nyabadza, F., Njagarah, J.B.H., Smith, R.J.: Modelling the dynamics of crystal meth ('tik') abuse in the presence of drug-supply chain in South Africa. Bull. Math. Biol. 75, 24-28 (2013)

23. Mushanyu, J., Nyabadza, F., Stewart, A.G.R.: Modelling the trends of inpatient and outpatient rehabilitation for methamphetamine in the Western Cape province of South Africa. BMC Res. Notes 8, Article ID 797 (2015)

24. Liu, P.Y., Zhang, L., Xing, Y.F.: Modelling and stability of a synthetic drugs transmission model with relapse and treatment. J. Appl. Math. Comput. 60, 465-484 (2019)

25. Ma, M.J., Liu, S.Y., Xiang, H., Li, J.: Dynamics of synthetic drugs transmission model with psychological addicts and general incidence rate. Physica A 491, 641-649 (2018)

26. Kundu, S., Maitra, S.: Dynamics of a delayed predator-prey system with stage structure and cooperation for preys. Chaos Solitons Fractals 114, 453-460 (2018) 
27. Keshri, N., Mishra, B.K.: Two time-delay dynamic model on the transmission of malicious signals in wireless sensor network. Chaos Solitons Fractals 68, 151-158 (2014)

28. Meng, X.Y., Wang, J.G.: Analysis of a delayed diffusive model with Beddington-DeAngelis functional response. Int. J. Biomath. 12, Article ID 1950047 (2019)

29. Zhang, Z.Z., Wang, Y.G.: Hopf bifurcation of a heroin model with time delay and saturated treatment function. Adv. Differ. Equ. 2019, Article ID 64 (2019)

30. Ren, J.G., Yang, X.F., Yang, L.X., Xu, Y.H., Yang, F.Z.: A delayed computer virus propagation model and its dynamics. Chaos Solitons Fractals 45, 74-79 (2012)

31. Driessche, P.V.D., Watmough, J.: Further notes on the basic reproduction number. In: Mathematical Epidemiology, pp. 159-178 (2008)

32. Liu, J.: Bifurcation analysis of a delayed predator-prey system with stage structure and Holling-ll functional response. Adv. Differ. Equ. 2015, Article ID 208 (2015)

33. Hassard, B.D., Kazarinoff, N.D., Wan, Y.H.: Theory and Applications of Hopf Bifurcation. Cambridge University Press, Cambridge (1981)

34. Peng, M., Zhang, Z.D., Wang, X.D.: Hybrid control of Hopf bifurcation in a Lotka-Volterra predator-prey model with two delays. Adv. Differ. Equ. 2017, Article ID 387 (2017)

35. Bianca, C., Ferrara, M., Guerrini, L.: The Cai model with time delay: existence of periodic solutions and asymptotic analysis. Appl. Math. Inf. Sci. 7, 21-27 (2013)

36. Xu, C.J.: Delay-induced oscillations in a competitor-competitor-mutualist Lotka-Volterra model. Complexity 2017, Article ID 2578043 (2017)

37. Meng, X.Y., Huo, H.F., Zhang, X.B., Xiang, H.: Stability and Hopf bifurcation in a three species system with feedback delays. Nonlinear Dyn. 64, 349-364 (2011)

38. Li, C.R.: A study on time-delay rumor propagation model with saturated control function. Adv. Differ. Equ. 2017, Article ID 255 (2017)

\section{Submit your manuscript to a SpringerOpen ${ }^{\circ}$ journal and benefit from:}

- Convenient online submission

- Rigorous peer review

- Open access: articles freely available online

- High visibility within the field

Retaining the copyright to your article

Submit your next manuscript at $\gg$ springeropen.com 